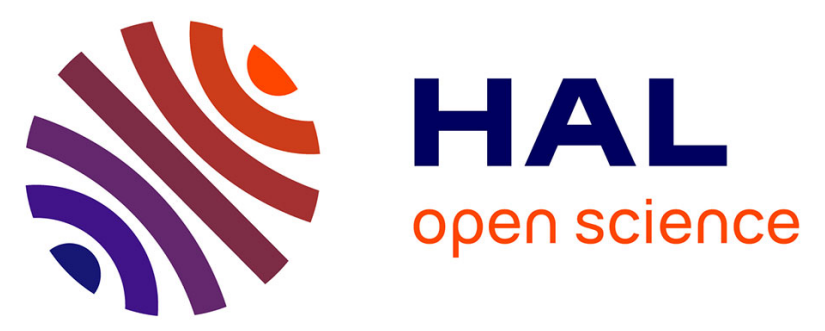

\title{
Observability and measurement allocation analysis for networked systems
}

Taha Boukhobza, Frédéric Hamelin

\section{To cite this version:}

Taha Boukhobza, Frédéric Hamelin. Observability and measurement allocation analysis for networked systems. 1st IFAC Workshop on Estimation and Control of Networked Systems, NecSys'09, Sep 2009, Venise, Italy. pp.108-113. hal-00422113

\section{HAL Id: hal-00422113 \\ https://hal.science/hal-00422113}

Submitted on 5 Oct 2009

HAL is a multi-disciplinary open access archive for the deposit and dissemination of scientific research documents, whether they are published or not. The documents may come from teaching and research institutions in France or abroad, or from public or private research centers.
L'archive ouverte pluridisciplinaire HAL, est destinée au dépôt et à la diffusion de documents scientifiques de niveau recherche, publiés ou non, émanant des établissements d'enseignement et de recherche français ou étrangers, des laboratoires publics ou privés. 


\title{
Observability and measurement allocation analysis for networked systems
}

\author{
Taha Boukhobza* Frédéric Hamelin * \\ * Centre de Recherche en Automatique de Nancy, Nancy-University, CNRS \\ UMR 7039, BP-70239, 54506 Vandouvre-lès-Nancy, France; (e-mail: \\ taha.boukhobza@cran.uhp-nancy.fr)
}

\begin{abstract}
This paper deals with the strong state observability analysis for Networked Systems which are composed of interconnected subsystems that exchange data over communication networks. More precisely, we interest to determine which data or measurements must arrive via the network to a subsystem in order to ensure that a local observer can estimate its state vector. The proposed approach is based on a graph-theoretic approach and assumes only the knowledge of the system's structure. It is suited to study large scale distributed systems because based on classical and well-known graph theory algorithms, which have polynomial complexity orders.

Copyright ${ }^{\circledR} 2009$ IFAC
\end{abstract}

Keywords: Networked Systems, strong state observability, measurements allocation, graph theory.

\section{INTRODUCTION}

Networked Control systems (NCS) are in general composed of a large number of interconnected devices or subsystems that exchange data through communication networks. Examples include industrial automation, building supervision, automotive control, ... NCSs provide many advantages such as modular and flexible system design, fast implementation, distribution. The observability of the internal state of each subsystem is one of the main properties which is strongly linked to the configuration of the distributed subsystems and to the data they exchange. Indeed, even if the global system is observable, when we subdivide it into several subsystems, the latter may be not structurally observable and in this case, it may be impossible to achieve the state estimation with a local observer. This can be quite awkward since the state estimate is useful in the control law synthesis, supervision, fault detection and so on. Thus, an analysis of the observability of the distributed system, in function of the informations exchanged on the network, is important for the observer design and so in the general conception of the system. Many studies use unknown input observers to deal with the observation of decentralized systems even if it is not in the context of Networked Control Systems. In this way, in the early 70's, Sanders et al. [1974] propose, under some decoupling assumptions, the design of a filter for interconnected dynamical systems in which the information pattern is decentralized. In Saif and Guan [1992], the authors propose a method for the design of decentralized reduced state estimator for large scale systems composed by interconnected systems using unknown input observers under some "matching condition". Also on the basis of unknown input observers, in Hou and Müller [1994], decentralized state function observer are designed for large scale interconnected systems. In the latter papers as in most others, the proposed approaches to address the observability or on decentralized systems deal with algebraic and geometric tools. The use of such tools requires the exact knowledge of the state space matrices characterizing the system's model. However, in many modeling problems, only zero entries of these matrices, which are determined by the physical laws, are fixed while the remaining entries are not precisely known. To study the properties of these systems in spite of poor knowledge we have on them, the idea is that we only keep the zero/non-zero entries in the state space matrices. Thus, we consider models where the fixed zeros are conserved while the non-zero entries are replaced by free parameters. The analysis of such systems, called structured systems, requires a low computational burden which allows one to deal with large scale systems. Many studies on structured systems are related to the graph-theoretic approach to analyse some system properties such as controllability, observability or the solvability of several classical control problems including disturbance rejection, input-output decoupling, .... These works are reviewed in the survey (Dion et al. [2003]) from which it results that the graph-theoretic approach provides simple, efficient and elegant solutions.

In this context, the state and input observability conditions provided in Boukhobza et al. [2007] for a linear systems with unknown inputs are a good point of departure to enhance the observability of Networked Control Systems. Besides, we address the problem of measurement allocation over the network. The paper is organised as follows: after Section 2, which is devoted to the problem formulation, a digraph representation of Networked Control systems is given in Section 3. The main result is enounced in Section 4. Finally, a conclusion ends the paper.

\section{PROBLEM STATEMENT}

In this paper, we consider networked systems having the numerically non-specified following model:

$$
\Sigma_{\Lambda}:\left\{\begin{array}{l}
\dot{x}=A^{\lambda} x+B^{\lambda} u \\
y=C^{\lambda} x+D^{\lambda} u
\end{array}\right.
$$

where $x \in \mathbb{R}^{n}, u \in \mathbb{R}^{q}$ and $y \in \mathbb{R}^{p}$ are respectively the state vector, the unknown input vector and the output vector. $A^{\lambda}$, $B^{\lambda}, C^{\lambda}$ and $D^{\lambda}$ represent matrices which elements are either fixed to zero or assumed to be nonzero free parameters noted $\lambda_{i}$. These parameters forms a vector $\Lambda=\left(\lambda_{1}, \ldots, \lambda_{h}\right)^{T} \in \mathbb{R}^{h}$. 
If all parameters $\lambda_{i}$ are numerically fixed, we obtain a so-called admissible realization of structured system $\Sigma_{\Lambda}$. We say that a property is true generically if it is true for almost all the realizations of structured system $\Sigma_{\Lambda}$. Here, "for almost all the realizations" is to be understood as "for all parameter values $\left(\Lambda \in \mathbb{R}^{h}\right)$ except for those in some proper algebraic variety in the parameter space". The proper algebraic variety for which the property is not true is the zero set of some nontrivial polynomial with real coefficients in the $h$ system parameters $\lambda_{1}, \lambda_{2}, \ldots, \lambda_{h}$.

Consider that $\Sigma_{\Lambda}$ is a networked distributed system constituted of several subsystems $\Sigma_{i}^{R}, i=1, \ldots, N$. Each subsystem satisfies to a model of the form:

$$
\Sigma_{i}^{R}\left\{\begin{array}{l}
\dot{x}_{i}=A_{i}^{\lambda} x_{i}+B_{i}^{\lambda} u_{i}+\sum_{j=1, j \neq i}^{N}\left(A_{i, j}^{\lambda} x_{j}+B_{i, j}^{\lambda} u_{j}\right) \\
y_{i}=C_{i}^{\lambda} x_{i}+D_{i}^{\lambda} u_{i}
\end{array}\right.
$$

where for $i=1, \ldots, N, x_{i} \in \mathbb{R}^{n_{i}}, y_{i} \in \mathbb{R}^{m_{i}}$ and $u_{i} \in \mathbb{R}^{p_{i}}$ represent respectively the state vector, the local measurement vector and the local input vector of subsystem $\Sigma_{i}^{R}$. Obviously, the whole state vector of system $\Sigma_{\Lambda}$ is constituted by the aggregation of the state vectors of subsystems $\Sigma_{i}^{R}, i=1, \ldots, N$ i.e. $x=\left(x_{1}^{T}, \ldots, x_{N}^{T}\right)^{T}$. Similarly, the whole input vector is $u=\left(u_{1}^{T}, \ldots, u_{N}^{T}\right)^{T}$ and the whole output vector is $y=$ $\left(y_{1}^{T}, \ldots, y_{N}^{T}\right)^{T}$. If a state or an input component of subsystem $\Sigma_{j}^{R}$ acts on the dynamics of another subsystem $\Sigma_{i}^{R}(i \neq j)$, we consider this component "as an unknown input" for subsystem $\Sigma_{i}^{R}$. Even if its dynamics has a known expression in the global model, it won't be implemented in the local observers used to reconstruct the state vector of each subsystems. In fact, this consideration is more pertinent and realistic from a practical point of view because implementing the whole system's model in each local observer can be, on the one hand, tedious and unpracticable and on the other hand does not allow to benefit from advantages of the subdivision of a large scale system into several smaller subsystems. Moreover, as we are interested in the observability problem, by the term "output" we mean here measurements. Otherwise, without loss of generality and for the sake of homogeneity, all the inputs are assumed to be unknown. Concerning the control input components, which are known, they are considered to be measured.

In the framework of this paper, the subsystems can exchange some informations through a network We consider without loss of generality that the measurements arriving to each subsystem $\Sigma_{i}^{R}$ are related to other subsystems $\Sigma_{j}^{R}(i \neq j)$ and can be modeled in the most general form as:

$$
\tilde{y}_{i}=\sum_{j=1, j \neq i}^{N} \tilde{C}_{i, j}^{\lambda} x_{j}(t)+\tilde{D}_{i, j}^{\lambda} u_{j}(t)
$$

The aim is to study the generic strong state observability (Trentelman et al. [2001]) of structured subsystems constituting $\Sigma_{\Lambda}$. This property is necessary and sufficient to ensure that the state components can be deduced from only the knowledge of the available measurements. First, we recall the definition of the generic strong state observability in the case of a structured linear system:

Definition 1. Structured linear system $\Sigma_{\Lambda}$ is generically strongly observable if, for almost all its realizations, $y(t)=0$ for $t \geq 0$ implies $x(t)=0$ for $t \geq 0$ whatever the unknown inputs $u(t)$.

Roughly speaking, the generic strong observability means that it is possible to the express the state of $\Sigma_{\Lambda}$ in function of the available measurements and their time derivatives.

In the framework of networked systems, we are interested in the generic strong observability of the state of each subsystem $\Sigma_{i}^{R}, i=1, \ldots, N$, and we consider two cases. In the first one, we assume that there are no external measurements arrive through the network to $\Sigma_{i}^{R}$. So, subsystem $\Sigma_{i}^{R}$ can only use its own measurement vector $y_{i}$ to estimate or reconstruct its state components. We call this case the decentralized autonomous observation scheme. In the second case, we assume that subsystem $\Sigma_{i}^{R}$ is linked to the network and can use the measurement vectors $y^{i}$ and $\tilde{y}^{i}$ to reconstruct its state components. We call this case the decentralized interconnected observation scheme. In order to handle these notions of local strong observability in the two previously described cases, we associate to each subsystem $\Sigma_{R}^{i}$, the following system:

$$
\Sigma_{i}^{R A}\left\{\begin{array}{l}
\dot{x}_{i}=A_{i}^{\lambda} x_{i}+B_{i}^{\lambda} u_{i}+\sum_{j=1, j \neq i}^{N} \tilde{B}_{i, j}^{\lambda} \tilde{u}_{j} \\
y_{i}=C_{i}^{\lambda} x_{i}+D_{i}^{\lambda} u_{i}
\end{array}\right.
$$

where for $i, j=1, \ldots, N, j \neq i$, unknown input $\tilde{u}_{j}=$ $\left(x_{j}^{T}, u_{j}^{T}\right)$ represents the interactions due to other subsystems and so matrix $\tilde{B}_{i, j}^{\lambda}=\left(A_{i, j}^{\lambda} B_{i, j}^{\lambda}\right)$. System $\Sigma_{i}^{R A}$ is equivalent to system $\Sigma_{R}^{i}$ by considering that vectors $x_{j}$ and $u_{j}, j \neq i$ are unknown inputs for subsystem $\Sigma_{i}^{R}$. We define now the state strong observability, relatively to these considered observation schemes, as follows:

Definition 2. Consider linear structured system $\Sigma_{\Lambda}$ constituted of several subsystems $\Sigma_{i}^{R}, i=1, \ldots, N$.

We say that that state vector $x_{i}(t)$ is generically locally strongly observable in a decentralized autonomous observation scheme if for system $\Sigma_{i}^{R A}$, having $y_{i}(t)=0$ for $t \geq 0$ implies $x_{i}(t)=0, \forall t \geq 0$ whatever the unknown inputs $u_{i}(t)$ and $\tilde{u}_{j}(t), j \neq i$.

Similarly, state $x_{i}(t)$ is generically strongly observable in a decentralized interconnected observation scheme if for system $\Sigma_{i}^{R A}$, having $y_{i}(t)=0$ and $\tilde{y}^{i}(t)=0$ for $t \geq 0$ implies that $x_{i}(t)=0, \forall t \geq 0$ whatever the unknown inputs $u_{i}(t)$ and $\tilde{u}_{j}(t), j \neq i$.

The aim of this paper is firstly to characterize graphically the strong observability of the each subsystems $\Sigma_{i}^{R}$ for both the considered observation schemes. Then, assuming that each subsystem is strongly observable if it is not submitted to external physical actions from other subsystems, we study the problem of data distribution over the network.

\section{GRAPH REPRESENTATION OF STRUCTURED LINEAR SYSTEMS}

\subsection{Digraph definition for structured linear system}

To structured system $\Sigma_{\Lambda}$ constituted by subsystems $\Sigma_{i}^{R}, i=$ $1, \ldots, N$, we associate a digraph noted $\mathcal{G}\left(\Sigma_{\Lambda}\right)$ which is constituted by a vertex set $\mathcal{V}$ and an edge set $\mathcal{E}$. More precisely, $\mathcal{V}=\bigcup_{i=1}^{N}\left(\mathbf{X}_{\mathbf{i}} \cup \mathbf{U}_{\mathbf{i}} \cup \mathbf{Y}_{\mathbf{i}} \cup \widetilde{\mathbf{Y}}_{\mathbf{i}}\right)$, where $\mathbf{X}_{\mathbf{i}}=$ $\left\{\mathbf{x}_{\mathbf{i}, \mathbf{1}}, \ldots, \mathbf{x}_{\mathbf{i}, \mathbf{n}_{\mathbf{i}}}\right\}$ is the set of state vertices for subsystem $i$,

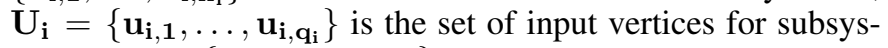
tem $i, \mathbf{Y}_{\mathbf{i}}=\left\{\mathbf{y}_{\mathbf{i}, \mathbf{1}}, \ldots, \mathbf{y}_{\mathbf{i}, \mathbf{p}_{\mathbf{i}}}\right\}$ is the set of output vertices for subsystem $i, \tilde{\mathbf{Y}}_{\mathbf{i}}=\left\{\tilde{\mathbf{y}}_{\mathbf{i}, \mathbf{1}}, \ldots, \tilde{\mathbf{y}}_{\left.\mathbf{i}, \tilde{\mathbf{p}}_{\mathbf{i}}\right\}}\right\}$ is the set of output vertices associated to the measurements arriving through the network to 
subsystem $i$. The edge set is

$\mathcal{E}=\bigcup_{i=1}^{N}\left(A_{i}\right.$-edges $\cup B_{i}$-edges $\cup C_{i}$-edges $\cup D_{i}$-edges

$\bigcup_{j=1, j \neq i}^{N}\left(A_{i, j}\right.$-edges $\cup B_{i, j}$-edges $\cup \tilde{C}_{i, j}$-edges $\cup \tilde{D}_{i, j}$-edges $\left.)\right)$,

where

$A_{i}$-edges $=\left\{\left(\mathbf{x}_{\mathbf{i}, \mathbf{j}}, \mathbf{x}_{\mathbf{i}, \mathbf{k}}\right) \mid A_{i}^{\lambda}(k, j) \neq 0\right\}$,

$B_{i}$-edges $=\left\{\left(\mathbf{u}_{\mathbf{i}, \mathbf{h}}, \mathbf{x}_{\mathbf{i}, \ell}\right) \mid B_{i}^{\lambda}(\ell, h) \neq 0\right\}$,

for $j \neq i, A_{i, j}$-edges $=\left\{\left(\mathbf{x}_{\mathbf{j}, \ell}, \mathbf{x}_{\mathbf{i}, \mathbf{h}}\right) \mid A_{i, j}^{\lambda}(h, \ell) \neq 0\right\}$,

$B_{i, j}$-edges $=\left\{\left(\mathbf{u}_{\mathbf{j}, \ell}, \mathbf{x}_{\mathbf{i}, \mathbf{h}}\right) \mid B_{i, j}^{\lambda}(h, \ell) \neq 0\right\}$,

$C_{i}$-edges $=\left\{\left(\mathbf{x}_{\mathbf{i}, \mathbf{h}}, \mathbf{y}_{\mathbf{i}, \ell}\right) \mid C_{i}^{\lambda}(\ell, h) \neq 0\right\}$,

$D_{i}$-edges $=\left\{\left(\mathbf{u}_{\mathbf{i}, \mathbf{h}}, \mathbf{y}_{\mathbf{i}, \ell}\right) \mid D_{i}^{\lambda}(\ell, h) \neq 0\right\}$,

for $j \neq i, \tilde{C}_{i, j}$-edges $=\left\{\left(\mathbf{x}_{\mathbf{j}, \ell}, \tilde{\mathbf{y}}_{\mathbf{i}, \mathbf{h}}\right) \mid \tilde{C}_{i, j}^{\lambda}(h, \ell) \neq 0\right\}$

and $\tilde{D}_{i, j}$-edges $=\left\{\left(\mathbf{u}_{\mathbf{j}, \ell}, \tilde{\mathbf{y}}_{\mathbf{i}, \mathbf{h}}\right) \mid \tilde{D}_{i, j}^{\lambda}(h, \ell) \neq 0\right\}$.

Here, $M(i, j)$ is the $(i, j)$ th element of matrix $M$ and $\left(\mathbf{v}_{\mathbf{1}}, \mathbf{v}_{\mathbf{2}}\right)$ denotes a directed edge from vertex $\mathbf{v}_{\mathbf{1}} \in \mathcal{V}$ to vertex $\mathbf{v}_{\mathbf{2}} \in \mathcal{V}$.

Example 3. Consider the following structured distributed system constituted of three subsystems:

Subsystem 1:

$A_{1}^{\lambda}=\left(\begin{array}{cccccc}0 & \lambda_{1} & 0 & 0 & 0 & 0 \\ 0 & 0 & 0 & 0 & 0 & 0 \\ 0 & 0 & \lambda_{2} & 0 & 0 & 0 \\ 0 & 0 & 0 & 0 & 0 & \lambda_{3} \\ 0 & 0 & 0 & 0 & \lambda_{4} & 0 \\ 0 & \lambda_{5} & 0 & 0 & 0 & 0\end{array}\right), A_{1,2}^{\lambda}=\left(\begin{array}{ccc}0 & 0 & 0 \\ \lambda_{6} & 0 & 0 \\ 0 & 0 & 0 \\ 0 & 0 & 0 \\ 0 & 0 & 0 \\ 0 & 0 & 0\end{array}\right), A_{1,3}^{\lambda}=\left(\begin{array}{cc}0 & 0 \\ 0 & \lambda_{7} \\ 0 & 0 \\ 0 & 0 \\ 0 & 0 \\ 0 & 0\end{array}\right)$,

$B_{1}^{\lambda}=\left(\begin{array}{c}0 \\ \lambda_{8} \\ 0 \\ 0 \\ 0 \\ 0\end{array}\right), B_{1,2}^{\lambda}=\left(\begin{array}{c}0 \\ 0 \\ 0 \\ 0 \\ 0 \\ \lambda_{9}\end{array}\right), C_{1}^{\lambda}=\left(\begin{array}{cccccc}\lambda_{10} & 0 & \lambda_{11} & 0 & 0 & 0 \\ 0 & 0 & 0 & \lambda_{12} & \lambda_{13} & 0 \\ 0 & 0 & \lambda_{14} & 0 & 0 & 0\end{array}\right)$,

$B_{1,3}^{\lambda}=0$ and $D_{1}^{\lambda}=0$.

Subsystem 2:

$A_{2}^{\lambda}=\left(\begin{array}{ccc}0 & \lambda_{15} & 0 \\ 0 & 0 & 0 \\ 0 & 0 & \lambda_{16}\end{array}\right), A_{2,1}^{\lambda}=A_{2,3}=0, B_{2}^{\lambda}=\left(\begin{array}{c}0 \\ \lambda_{17} \\ \lambda_{18}\end{array}\right), B_{2,1}^{\lambda}=B_{2,3}^{\lambda}=$ $0, C_{2}^{\lambda}=\left(\begin{array}{ccc}\lambda_{19} & 0 & 0 \\ 0 & 0 & \lambda_{20}\end{array}\right)$ and $D_{2}^{\lambda}=\left(\begin{array}{c}0 \\ \lambda_{21}\end{array}\right)$.

Subsystem 3:

$A_{3}^{\lambda}=\left(\begin{array}{cc}0 & \lambda_{22} \\ 0 & 0\end{array}\right), A_{3,1}^{\lambda}=0, A_{3,2}^{\lambda}=\left(\begin{array}{ccc}0 & 0 & \lambda_{23} \\ 0 & 0 & \lambda_{24}\end{array}\right), B_{3}^{\lambda}=\left(\begin{array}{c}0 \\ \lambda_{25}\end{array}\right)$, $B_{3,1}^{\lambda}=B_{3,2}^{\lambda}=0, C_{3}^{\lambda}=\left(\begin{array}{cc}\lambda_{26} & 0 \\ 0 & 0\end{array}\right)$ and $D_{3}^{\lambda}=\left(\begin{array}{c}0 \\ \lambda_{27}\end{array}\right)$.

To such a model, we associate the digraph of figure 1 . For a sake of simplicity in this example, the set of output vertices associated to the measurements $\tilde{Y}$ arriving through the network to each subsystem are not represented.

\subsection{Notations and definitions}

- Two edges $e_{1}=\left(\mathbf{v}_{\mathbf{1}}, \mathbf{v}_{\mathbf{1}}^{\prime}\right)$ and $e_{2}=\left(\mathbf{v}_{\mathbf{2}}, \mathbf{v}_{\mathbf{2}}^{\prime}\right)$ are $v$-disjoint if $\mathbf{v}_{\mathbf{1}} \neq \mathbf{v}_{\mathbf{2}}$ and $\mathbf{v}_{\mathbf{1}}^{\prime} \neq \mathbf{v}_{\mathbf{2}}^{\prime}$. Some edges are $v$-disjoint if they are mutually $v$-disjoint.

- Path $P$ containing vertices $\mathbf{v}_{\mathbf{r}_{\mathbf{0}}}, \ldots, \mathbf{v}_{\mathbf{r}_{\mathbf{i}}}$ is denoted $P=$ $\mathbf{v}_{\mathbf{r}_{\mathbf{0}}} \rightarrow \mathbf{v}_{\mathbf{r}_{1}} \rightarrow \ldots \rightarrow \mathbf{v}_{\mathbf{r}_{\mathbf{i}}}$, where $\left(\mathbf{v}_{\mathbf{r}_{\mathbf{j}}}, \mathbf{v}_{\mathbf{r}_{\mathbf{j}+1}}\right) \in \mathcal{E}$ for $j=0,1 \ldots, i-1$. We say that $P$ covers $\mathbf{v}_{\mathbf{r}_{0}}, \mathbf{v}_{\mathbf{r}_{1}}, \ldots, \mathbf{v}_{\mathbf{r}_{\mathbf{i}}}$.

- A path is simple when every vertex occurs only once in this path. Some paths are disjoint if they have no common vertex. Path $P$ is a $\mathbf{Y}$-topped path if its end vertex belongs to $\mathbf{Y}$.

Let $\mathcal{V}_{1}$ and $\mathcal{V}_{2}$ denote two subsets of $\mathcal{V}$.

- The cardinality of $\mathcal{V}_{1}$ is noted card $\left(\mathcal{V}_{1}\right)$.

- $\theta\left(\mathcal{V}_{1}, \mathcal{V}_{2}\right)$ is the maximal number of $v$-disjoint edges from $\mathcal{V}_{1}$ to $\mathcal{V}_{2}$.

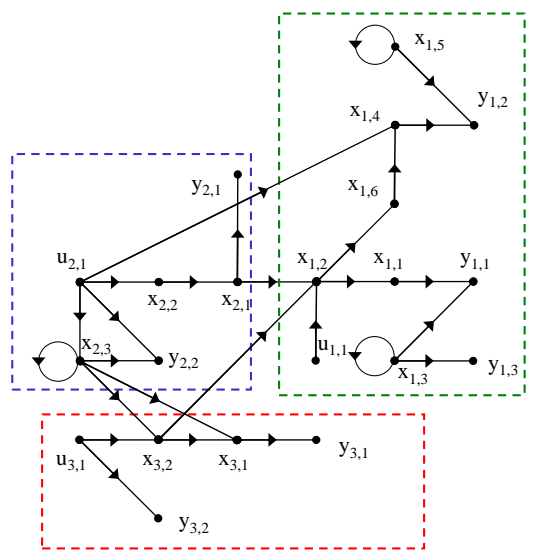

Figure 1. Digraph associated to Example 3

- A path $P$ is said a $\mathcal{V}_{1}-\mathcal{V}_{2}$ path if its begin vertex belongs to $\mathcal{V}_{1}$ and its end vertex belongs to $\mathcal{V}_{2}$. If the only vertices of $P$ belonging to $\mathcal{V}_{1} \cup \mathcal{V}_{2}$ are its begin and its end vertices, $P$ is said a direct $\mathcal{V}_{1}-\mathcal{V}_{2}$ path.

- A set of $\ell$ disjoint $\mathcal{V}_{1}-\mathcal{V}_{2}$ paths is called a $\mathcal{V}_{1}-\mathcal{V}_{2}$ linking of size $\ell$. The linkings, which consist of a maximal number of disjoint $\mathcal{V}_{1}-\mathcal{V}_{2}$ paths, are called maximum $\mathcal{V}_{1}-\mathcal{V}_{2}$ linkings. We define by $\rho\left(\mathcal{V}_{1}, \mathcal{V}_{2}\right)$ the size of these maximum $\mathcal{V}_{1}-\mathcal{V}_{2}$ linkings.

- $V_{\text {ess }}\left(\mathcal{V}_{1}, \mathcal{V}_{2}\right) \stackrel{\text { def }}{=}\{\mathbf{v} \in \mathcal{V} \mid \mathbf{v}$ is covered by every maximum $\mathcal{V}_{1}-\mathcal{V}_{2}$ linking $\}$

- $\mathrm{S} \subseteq \mathcal{V}$ is a separator between sets $\mathcal{V}_{1}$ and $\mathcal{V}_{2}$ if every path from $\mathcal{V}_{1}$ to $\mathcal{V}_{2}$ contains at least one vertex in $\mathbf{S}$. We call minimum separators between $\mathcal{V}_{1}$ and $\mathcal{V}_{2}$ any separators having the smallest size. According to Menger's Theorem, the latter is equal to $\rho\left(\mathcal{V}_{1}, \mathcal{V}_{2}\right)$.

- There exist two uniquely determined minimum separators between $\mathcal{V}_{1}$ and $\mathcal{V}_{2}$ noted $\mathbf{S}^{\mathbf{o}}\left(\mathcal{V}_{1}, \mathcal{V}_{2}\right)$ such that:

- $\mathbf{S}^{\mathbf{o}}\left(\mathcal{V}_{1}, \mathcal{V}_{2}\right)$ is the set of begin vertices of all direct $V_{\text {ess }}\left(\mathcal{V}_{1}, \mathcal{V}_{2}\right)$ $\mathcal{V}_{2}$ paths, where $V_{\text {ess }}\left(\mathcal{V}_{1}, \mathcal{V}_{2}\right) \cap \mathcal{V}_{2}$ is considered, in the present definition, as input vertices. Vertex subset $\mathbf{S}^{\mathbf{o}}\left(\mathcal{V}_{1}, \mathcal{V}_{2}\right)$ is called the minimum output separator.

- $\mathbf{S}^{\mathbf{i}}\left(\mathcal{V}_{1}, \mathcal{V}_{2}\right)$ is the set of begin vertices of all direct $\mathcal{V}_{1}$ $V_{\text {ess }}\left(\mathcal{V}_{1}, \mathcal{V}_{2}\right)$ paths, where $V_{\text {ess }}\left(\mathcal{V}_{1}, \mathcal{V}_{2}\right) \cap \mathcal{V}_{2}$ is considered, in the present definition, as input vertices. Vertex subset $\mathbf{S}^{\mathbf{i}}\left(\mathcal{V}_{1}, \mathcal{V}_{2}\right)$ is called the minimum input separator.

\section{MAIN RESULTS}

We first recall hereafter the graphic conditions which ensure the generic strong observability of a classical structured linear systems Boukhobza et al. [2007]:

Proposition 1. Structured linear system $\Sigma_{\Lambda}$ is generically strongly observable iff in its associated digraph $\mathcal{G}\left(\Sigma_{\Lambda}\right)$

Cond1. $\theta(\mathbf{X} \cup \mathbf{U}, \mathbf{X} \cup \mathbf{Y})=n+\theta(\mathbf{U}, \mathbf{X} \cup \mathbf{Y})$;

Cond2. every state vertex is the begin vertex of a $\mathbf{Y}$-topped path;

Cond3. $\left\{\mathbf{x}_{\mathbf{i}} \mid \rho\left(\mathbf{U} \cup\left\{\mathbf{x}_{\mathbf{i}}\right\}, \mathbf{Y}\right)=\rho(\mathbf{U}, \mathbf{Y})\right\} \subseteq V_{\text {ess }}(\mathbf{U}, \mathbf{Y} \cup \mathbf{X})$.

\subsection{Strong observability conditions for networked systems}

Let us now consider that the structured linear system $\Sigma_{\Lambda}$ is a distributed system and constituted of several subsystems $\Sigma_{i}^{R}$, $i=1, \ldots, N$. We apply the previous result in order to obtain the strong observability conditions of a given subsystem $\Sigma_{i}^{R}$ for both decentralized autonomous and decentralized interconnected observation schemes. We can consider for subsystem 
$\Sigma_{i}^{R}$ that the unknown input set is constituted by its own unknown input set plus all the state and unknown components related to the other subsystems $\Sigma_{j}^{R}, j=1, \ldots, i-1, i+$ $1, \ldots, N$ which act on $\Sigma_{i}^{R}$. Let us denote by $\tilde{\mathbf{U}}_{\mathbf{i}}=\{\mathbf{v} \in$ $\bigcup_{j=1, j \neq i}^{N}\left(\mathbf{U}_{\mathbf{j}} \cup \mathbf{X}_{\mathbf{j}}\right)$ such that $\left.\theta\left(\{\mathbf{v}\}, \mathbf{X}_{\mathbf{i}}\right) \neq 0\right\}$ and $\overline{\mathbf{U}}_{\mathbf{i}}=\tilde{\mathbf{U}}_{\mathbf{i}} \cup$ $\mathbf{U}_{\mathbf{i}}$. Thus, when we study subsystem $\Sigma_{i}^{R}$, we consider in $\mathcal{G}\left(\Sigma_{\Lambda}\right)$ that all the edges ending by an element of $\tilde{\mathbf{U}}_{\mathbf{i}}$ are removed. This digraph is similar to the one of $\Sigma_{i}^{R A}$.

When we deal with the autonomous scheme, only the measurements done on $\Sigma_{i}^{R}$ are available for the local observer dedicated to $\Sigma_{i}^{R}$. Thus, we deduce from Proposition 1:

Proposition 2. Consider structured system $\Sigma_{\Lambda}$ represented by digraph $\mathcal{G}\left(\Sigma_{\Lambda}\right)$ and constituted by subsystems $\Sigma_{i}^{R}, i=$ $1, \ldots, N$. Subsystem $\Sigma_{i}^{R}$ is generically strongly observable in an autonomous scheme iff in its associated digraph $\mathcal{G}_{i}$

Cond1. $\theta\left(\mathbf{X}_{\mathbf{i}} \cup \overline{\mathbf{U}}_{\mathbf{i}}, \mathbf{X}_{\mathbf{i}} \cup \mathbf{Y}_{\mathbf{i}}\right)=n_{i}+\theta\left(\overline{\mathbf{U}}_{\mathbf{i}} \cup \mathbf{X}_{\mathbf{i}}, \mathbf{X}_{\mathbf{i}} \cup \mathbf{Y}_{\mathbf{i}}\right)$ (maximal matching condition).

Cond2. every state vertex in $\mathbf{X}_{\mathbf{i}}$ is the begin vertex of a $\mathbf{Y}_{\mathbf{i}^{-}}$ topped path (output connectivity condition);

Cond3. $\left\{\mathbf{x}_{\ell} \in \mathbf{X}_{\mathbf{i}} \mid \rho\left(\overline{\mathbf{U}}_{\mathbf{i}} \cup\left\{\mathbf{x}_{\ell}\right\}, \mathbf{Y}_{\mathbf{i}}\right)=\rho\left(\overline{\mathbf{U}}_{\mathbf{i}}, \mathbf{Y}_{\mathbf{i}}\right)\right\} \subseteq V_{\text {ess }}\left(\overline{\mathbf{U}}_{\mathbf{i}}, \mathbf{Y}_{\mathbf{i}}\right)$ (length condition).

Concerning the subsystems of Example 3, we have:

Subsystem 1 has 6 state components $\left(n_{1}=6\right)$. It is submitted to one internal unknown input: $\mathbf{u}_{\mathbf{1}, \mathbf{1}}$ and three external inputs: $\mathbf{x}_{\mathbf{2}, \mathbf{1}}, \mathbf{u}_{\mathbf{2}, \mathbf{1}}$ from Subsystem 2 and $\mathbf{x}_{\mathbf{3}, \mathbf{2}}$ from Subsystem 3 and so $\overline{\mathbf{U}}_{\mathbf{1}}=\left\{\mathbf{u}_{1,1}, \mathbf{x}_{2,1}, \mathbf{u}_{2,1}, \mathbf{x}_{\mathbf{3}, \mathbf{2}}\right\}, \mathbf{X}_{\mathbf{1}}=$ $\left\{\mathrm{x}_{1,1}, \mathrm{x}_{1,2}, \mathrm{x}_{1,3}, \mathrm{x}_{1,4}, \mathrm{x}_{1,5}, \mathrm{x}_{1,6}\right\}, \mathrm{Y}_{1}=\left\{\mathrm{y}_{1,1}, \mathrm{y}_{1,2}, \mathrm{y}_{1,3}\right\}$ We have that $\theta\left(\overline{\mathbf{U}}_{\mathbf{1}}, \mathbf{X}_{\mathbf{1}} \cup \mathbf{Y}_{\mathbf{1}}\right)=2$ and $\theta\left(\overline{\mathbf{U}}_{\mathbf{1}} \cup \mathbf{X}_{\mathbf{1}}, \mathbf{X}_{\mathbf{1}} \cup\right.$ $\left.\mathbf{Y}_{\mathbf{1}}\right)=7 \neq n_{1}+\theta\left(\overline{\mathbf{U}}_{\mathbf{1}}, \mathbf{X}_{\mathbf{1}} \cup \mathbf{Y}_{\mathbf{1}}\right)=8$. Thus, the maximal matching condition is not satisfied and Subsystem 1 is not generically strongly observable in an autonomous observation scheme. Note that the output connectivity condition is satisfied. Finally, we have that $\mathbf{u}_{2,1}, \mathbf{x}_{1,1}, \mathbf{x}_{1,2}, \mathbf{x}_{1,4}, \mathbf{y}_{1,1}$ and $\mathbf{y}_{1,2}$ constitute $V_{\text {ess }}\left(\overline{\mathbf{U}}_{\mathbf{1}}, \mathbf{Y}_{\mathbf{1}}\right)$. On the other hand, $\rho\left(\overline{\mathbf{U}}_{\mathbf{1}} \cup\right.$ $\left.\left\{\mathbf{x}_{\mathbf{1}, \mathbf{5}}\right\}, \mathbf{Y}_{\mathbf{1}}\right)=\rho\left(\overline{\mathbf{U}}_{\mathbf{1}} \cup\left\{\mathbf{x}_{\mathbf{1}, \mathbf{6}}\right\}, \mathbf{Y}_{\mathbf{1}}\right)=\rho\left(\overline{\mathbf{U}}_{\mathbf{1}}, \mathbf{Y}_{\mathbf{1}}\right)=2$. Yet, neither $\mathrm{x}_{1,5}$ nor $\mathrm{x}_{1,6}$ are essential. Thus, the length condition is also not satisfied.

Subsystem 2 is not submitted to inputs from other subsystems. For such subsystem, $\mathbf{X}_{\mathbf{2}}=\left\{\mathbf{x}_{\mathbf{2}, \mathbf{1}}, \mathbf{x}_{\mathbf{2}, \mathbf{2}}, \mathbf{x}_{\mathbf{2}, \mathbf{3}}\right\}, \mathbf{Y}_{\mathbf{2}}=$ $\left\{\mathbf{y}_{2,1}, \mathbf{y}_{2,2}\right\}$ and $\overline{\mathbf{U}}_{2}=\mathbf{U}_{2}=\left\{\mathbf{u}_{2,1}\right\}$. We have that $\theta\left(\overline{\mathbf{U}}_{\mathbf{2}}, \mathbf{X}_{\mathbf{2}} \cup \mathbf{Y}_{\mathbf{2}}\right)=2$ and $\theta\left(\overline{\mathbf{U}}_{\mathbf{2}} \cup \mathbf{X}_{\mathbf{2}}, \mathbf{X}_{\mathbf{2}} \cup \mathbf{Y}_{\mathbf{2}}\right)=4=$ $n_{2}+\theta\left(\overline{\mathbf{U}}_{\mathbf{2}}, \mathbf{X}_{\mathbf{2}} \cup \mathbf{Y}_{\mathbf{2}}\right)$. Thus, the maximal matching condition is satisfied. Furthermore, the output connectivity condition is also satisfied. Finally, $\rho\left(\mathbf{U}_{\mathbf{2}}, \mathbf{Y}_{\mathbf{2}}\right)=1$ and $V_{\text {ess }}\left(\overline{\mathbf{U}}_{\mathbf{2}}, \mathbf{Y}_{\mathbf{2}}\right)=$ $\left\{\mathbf{u}_{\mathbf{2}, \mathbf{1}}\right\}$ but $\rho\left(\mathbf{U}_{\mathbf{2}} \cup\left\{\mathbf{x}_{\mathbf{2}, \mathbf{1}}\right\}, \mathbf{Y}_{\mathbf{2}}\right)=\rho\left(\mathbf{U}_{\mathbf{2}} \cup\left\{\mathbf{x}_{\mathbf{2}, \mathbf{2}}\right\}, \mathbf{Y}_{\mathbf{2}}\right)=2$, which implies that the length condition is also satisfied. Therefore Subsystem 2 is generically strongly observable in an autonomous scheme.

Subsystem 3 is submitted to one internal input: $\mathbf{u}_{\mathbf{3}, \mathbf{1}}$ and one

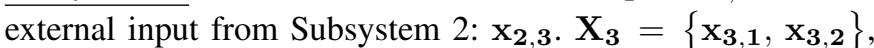
$\mathbf{Y}_{\mathbf{3}}=\left\{\mathbf{y}_{3,1}, \mathbf{y}_{\mathbf{3}, 2}\right\}, \overline{\mathbf{U}}_{\mathbf{3}}=\left\{\mathbf{u}_{3,1}, \mathbf{x}_{2,3}\right\}$. On the one hand, $\theta\left(\overline{\mathbf{U}}_{\mathbf{3}}, \mathbf{X}_{\mathbf{3}} \cup \mathbf{Y}_{\mathbf{3}}\right)=2$ and $\theta\left(\overline{\mathbf{U}}_{\mathbf{3}} \cup \mathbf{X}_{\mathbf{3}}, \mathbf{X}_{\mathbf{3}} \cup \mathbf{Y}_{\mathbf{3}}\right)=4=n_{3}+$ $\theta\left(\overline{\mathbf{U}}_{\mathbf{3}}, \mathbf{X}_{\mathbf{3}} \cup \mathbf{Y}_{\mathbf{3}}\right)$. Thus, the maximal matching condition is satisfied. Furthermore, the output connectivity condition is also satisfied. Moreover, $\rho\left(\overline{\mathbf{U}}_{\mathbf{3}}, \mathbf{Y}_{\mathbf{3}}\right)=2$ and $V_{\text {ess }}\left(\overline{\mathbf{U}}_{\mathbf{3}}, \mathbf{Y}_{\mathbf{3}}\right)=$ $\left\{\mathbf{u}_{\mathbf{3}, \mathbf{1}}, \mathbf{x}_{\mathbf{2}, \mathbf{3}}, \mathbf{x}_{\mathbf{3}, \mathbf{1}}, \mathbf{y}_{\mathbf{3}, \mathbf{1}}, \mathbf{y}_{\mathbf{3}, \mathbf{2}}\right\}$. Yet, $\rho\left(\overline{\mathbf{U}}_{\mathbf{3}} \cup\left\{\mathbf{x}_{\mathbf{3}, \mathbf{2}}\right\}, \mathbf{Y}_{\mathbf{3}}\right)=2$ and $\mathbf{x}_{\mathbf{3}, \mathbf{2}} \notin V_{\text {ess }}\left(\overline{\mathbf{U}}_{\mathbf{3}}, \mathbf{Y}_{\mathbf{3}}\right)$. Thus, the length condition is not satisfied and Subsystem 3.

In the next part of the paper we will interest in determining what external measurements must be transmitted via the network to a subsystem in order to make it strongly observable in a distributed interconnected observation scheme. At this aim, we first give the conditions of strong observability for this scheme: Proposition 3. Consider structured system $\Sigma_{\Lambda}$ represented by digraph $\mathcal{G}\left(\Sigma_{\Lambda}\right)$ and constituted by subsystems $\Sigma_{i}^{R}, i=$ $1, \ldots, N$. Subsystem $\Sigma_{i}^{R}$ is generically strongly observable in an interconnected (I.C.) scheme iff in its associated digraph $\mathcal{G}_{i}\left(\Sigma_{\Lambda}\right)$

Cond1. $\theta\left(\mathbf{X}_{\mathbf{i}} \cup \overline{\mathbf{U}}_{\mathbf{i}}, \mathbf{X}_{\mathbf{i}} \cup \mathbf{Y}_{\mathbf{i}} \cup \tilde{\mathbf{Y}}_{\mathbf{i}}\right)=n_{i}+\theta\left(\overline{\mathbf{U}}_{\mathbf{i}}, \mathbf{X}_{\mathbf{i}} \cup \mathbf{Y}_{\mathbf{i}} \cup \tilde{\mathbf{Y}}_{\mathbf{i}}\right)$ (I.C. maximal matching condition).

Cond2. every state vertex in $\mathbf{X}_{\mathbf{i}}$ is the begin vertex of a $\mathbf{Y}_{\mathbf{i}^{-}}$ topped path (output connectivity condition);

Cond3. $\left\{\mathbf{x}_{\ell} \in \mathbf{X}_{\mathbf{i}} \mid \rho\left(\overline{\mathbf{U}}_{\mathbf{i}} \cup\left\{\mathbf{x}_{\ell}\right\}, \mathbf{Y}_{\mathbf{i}} \cup \tilde{\mathbf{Y}}_{\mathbf{i}}\right)=\rho\left(\overline{\mathbf{U}}_{\mathbf{i}}, \mathbf{Y}_{\mathbf{i}} \cup\right.\right.$ $\left.\left.\tilde{\mathbf{Y}}_{\mathbf{i}}\right)\right\} \subseteq V_{e s s}\left(\overline{\mathbf{U}}_{\mathbf{i}}, \mathbf{Y}_{\mathbf{i}} \cup \tilde{\mathbf{Y}}_{\mathbf{i}}\right)$ (I.C. length condition).

Consider Example3, and assume that the measurement $\tilde{y}_{3,1}=$ $\mathbf{x}_{\mathbf{2}, \mathbf{3}}$ is transmitted to Subsystem 3. In this case, $V_{\text {ess }}\left(\overline{\mathbf{U}}_{\mathbf{3}}, \mathbf{Y}_{\mathbf{3}} \cup\right.$ $\left.\tilde{\mathbf{Y}}_{\mathbf{3}}\right)=\left\{\mathbf{u}_{\mathbf{3}, \mathbf{1}}, \mathbf{x}_{\mathbf{2}, 3}, \mathbf{y}_{\mathbf{3}, 2}\right\}$ and so the I.C. length condition is satisfied. Hence, Subsystem 3 is generically strongly observable in an interconnected scheme when it receives via the network the measurement $\tilde{y}_{3,1}=\mathbf{x}_{\mathbf{2}, \mathbf{3}}$.

\subsection{Necessary external measurements transmitted via network} for the strong observability

Let us assume that each subsystem is strongly locally observable or in other words that subsystems

$$
\left\{\begin{array}{l}
\dot{x}_{i}(t)=A_{i}^{\lambda} x_{i}(t)+B_{i}^{\lambda} u_{i}(t) \\
y_{i}(t)=C_{i}^{\lambda} x_{i}(t)+D_{i}^{\lambda} u_{i}(t)
\end{array} \quad i=1, \ldots, N\right.
$$

are strongly observable. If it is not the case, we must add some local measurements. This aspect has been already treated (Boukhobza and Hamelin [2009]) and have no major relevance for the present study because is not related to networked systems. In this case, the following conditions are true:

- $\theta\left(\mathbf{X}_{\mathbf{i}} \cup \mathbf{U}_{\mathbf{i}}, \mathbf{X}_{\mathbf{i}} \cup \mathbf{Y}_{\mathbf{i}}\right)=n_{i}+\theta\left(\mathbf{U}_{\mathbf{i}}, \mathbf{X}_{\mathbf{i}} \cup \mathbf{Y}_{\mathbf{i}}\right.$ (local maximal matching condition).

- every state vertex in $\mathbf{X}_{\mathbf{i}}$ is the begin vertex of a $\mathbf{Y}_{\mathbf{i}}$-topped path (output connectivity condition);

- $\left\{\mathbf{x}_{\ell} \in \mathbf{X}_{\mathbf{i}} \mid \rho\left(\mathbf{U}_{\mathbf{i}} \cup\left\{\mathbf{x}_{\ell}\right\}, \mathbf{Y}_{\mathbf{i}}\right)=\rho\left(\mathbf{U}_{\mathbf{i}}, \mathbf{Y}_{\mathbf{i}}\right)\right\} \subseteq V_{\text {ess }}\left(\mathbf{U}_{\mathbf{i}}, \mathbf{Y}_{\mathbf{i}}\right)$ (local length condition).

Note that all subsystems of Example 3 satisfy the conditions above and are strongly observable if they are not submitted to external inputs.

The aim of this subsection is to study what are the measurements which must be transmitted to subsystem $\Sigma_{i}^{R}$ in order to recover the strong observability in the distributed interconnected observation scheme. More precisely, we start from a subsystem which has enough local sensors in order to guarantee that it is strongly observable if it is not submitted to the physical interactions from other subsystems. Our objective is to provide, for each subsystem, the set of external measurements represented by $\tilde{\mathbf{Y}}_{\mathbf{i}}$ which allow to recover the conditions of Proposition 3, knowing that the output connectivity condition is already satisfied since each subsystem is strongly locally observable. To answer to this question, our proposed procedure consists of two steps which correspond one by one to conditions Cond1. and Cond3. of Proposition3. 


\subsection{Transmitted external measurements for the I.C. maximal matching condition}

The first objective of the measurement $\tilde{\mathbf{Y}}_{\mathbf{i}}$ arriving to subsystem $\Sigma_{i}^{R}$ is to obtain $\theta\left(\mathbf{X}_{\mathbf{i}} \cup \overline{\mathbf{U}}_{\mathbf{i}}, \mathbf{X}_{\mathbf{i}} \cup \mathbf{Y}_{\mathbf{i}} \cup \tilde{\mathbf{Y}}_{\mathbf{i}}\right)=n_{i}+\theta\left(\overline{\mathbf{U}}_{\mathbf{i}}, \mathbf{X}_{\mathbf{i}} \cup\right.$ $\left.\mathbf{Y}_{\mathbf{i}} \cup \tilde{\mathbf{Y}}_{\mathbf{i}}\right)$. This maximal matching condition is also equivalent to have $\forall \mathbf{V}_{\mathbf{1}} \subseteq \mathbf{X}_{\mathbf{i}} \cup \overline{\mathbf{U}}_{\mathbf{i}}$

$$
\begin{aligned}
& \theta\left(\mathbf{V}_{\mathbf{1}}, \mathbf{X}_{\mathbf{i}} \cup \mathbf{Y}_{\mathbf{i}} \cup \tilde{\mathbf{Y}}_{\mathbf{i}}\right)= \\
& \operatorname{card}\left(\mathbf{V}_{\mathbf{1}} \cap \overline{\mathbf{X}}_{\mathbf{i}}\right)+\theta\left(\mathbf{V}_{\mathbf{1}} \cap \overline{\mathbf{U}}_{\mathbf{i}}, \mathbf{X}_{\mathbf{i}} \cup \mathbf{Y}_{\mathbf{i}} \cup \tilde{\mathbf{Y}}_{\mathbf{i}}\right)
\end{aligned}
$$

When the latter condition is not satisfied for some vertex subset $\mathbf{V}_{1}$, we say that there is dilation in the digraph of the system. The aim of transmitted measures at this first stage is to eliminate all these dilations. As in Commault et al. [2008], we also use the Dulmage-Mendelsohn decomposition. Hence, we define a bipartite graph in order to localize the dilations occurring in the digraph of subsystem $\Sigma_{i}^{R}$. This bipartite graph is noted $B_{i}\left(\Sigma_{\Lambda}\right)=\left(V^{+}, V^{-}, W\right)$, where $V^{+}$and $V^{-}$are two disjoint vertex subsets and $W$ is the edge set. More precisely, $V^{+}=\mathbf{X}_{\mathbf{i}}^{+} \cup \overline{\mathbf{U}}_{\mathbf{i}}^{+}$and $V^{-}=\mathbf{Y}_{\mathbf{i}}^{-} \cup \mathbf{X}_{\mathbf{i}}^{-}$, with $\mathbf{X}_{\mathbf{i}}^{+}=\left\{\mathbf{x}_{\mathbf{i}, \mathbf{1}}^{+}, \mathbf{x}_{\mathbf{i}, \mathbf{2}}^{+}, \ldots, \mathbf{x}_{\mathbf{n}_{\mathbf{i}}, \mathbf{i}}^{+}\right\}, \overline{\mathbf{U}}_{\mathbf{i}}^{+}=\left\{\overline{\mathbf{u}}_{\mathbf{i}, \mathbf{1}}^{+}, \overline{\mathbf{u}}_{\mathbf{i}, \mathbf{2}}^{+}, \ldots, \overline{\mathbf{u}}_{\overline{\mathbf{q}}_{\mathbf{i}}, \mathbf{i}}^{+}\right\}$, $\mathbf{X}_{\mathbf{i}}^{-}=\left\{\mathbf{x}_{\mathbf{i}, \mathbf{1}}^{-}, \mathbf{x}_{\mathbf{i}, \mathbf{2}}^{-}, \ldots, \mathbf{x}_{\mathbf{i}, \mathbf{n}}^{-}\right\}, \mathbf{Y}_{\mathbf{i}}^{-}=\left\{\mathbf{y}_{\mathbf{i}, \mathbf{1}}^{-}, \mathbf{y}_{\mathbf{i}, \mathbf{2}}^{-}, \ldots, \mathbf{y}_{\mathbf{i}, \mathbf{p}}^{-}\right\}$. Edge set $W$ is defined such that $\left(\mathbf{v}_{\mathbf{i}}^{+}, \mathbf{v}_{\mathbf{j}}^{-}\right) \in W$ iff there exists an edge $\left(\mathbf{v}_{\mathbf{i}}, \mathbf{v}_{\mathbf{j}}\right)$ in the associated digraph $\mathcal{G}\left(\Sigma_{\Lambda}\right)$. A matching in a bipartite graph $B_{i}\left(\Sigma_{\Lambda}\right)=\left(V^{+}, V^{-}, W\right)$ is an edge set $M \subseteq W$ such that all the edges of $M$ are disjoint . A matching is maximal if it has a maximal cardinality which is equal to $\theta\left(V^{+}, V^{-}\right)$which is equal by construction of the digraph to $\theta\left(\mathbf{X}_{\mathbf{i}} \cup \overline{\mathbf{U}}_{\mathbf{i}}, \mathbf{X}_{\mathbf{i}} \cup \mathbf{Y}_{\mathbf{i}}\right)$. Then, the fact that condition Cond1. of Proposition 3 is not satisfied i.e. $\theta\left(\mathbf{V}^{+}, \mathbf{V}^{-}\right)<\operatorname{card}\left(\mathbf{X}_{\mathbf{i}}^{+}\right)+\theta\left(\overline{\mathbf{U}}_{\mathbf{i}}^{+}, \mathbf{V}^{-}\right)$, implies that some additional measurement vertices $\tilde{\mathbf{Y}}_{\mathbf{i}}$ are needed to complete $\mathbf{V}^{-}$. This number is less or equal to card $\left(\mathbf{V}^{+}\right)-\theta\left(\mathbf{V}^{+}, \mathbf{V}^{-}\right)$. In addition to determining the exact number of required transmitted measurements, another problem is to precise as finely as possible because obviously transmitting any data does not allow to recover the maximal matching condition. At this aim,let us associate to each maximal matching $M$, a non bipartite digraph noted $B_{M}\left(\Sigma_{\Lambda}\right)=\left(\mathbf{V}^{+}, \mathbf{V}^{-}, \bar{W}\right)$ where $\left(\mathbf{v}_{\mathbf{1}}, \mathbf{v}_{\mathbf{2}}\right) \in \bar{W} \Leftrightarrow$ $\left(\mathbf{v}_{\mathbf{1}}, \mathbf{v}_{\mathbf{2}}\right) \in W$ or $\left(\mathbf{v}_{\mathbf{2}}, \mathbf{v}_{\mathbf{1}}\right) \in M$.

We denote by $\partial^{+} \mathbf{M}\left(\right.$ resp. $\left.\partial^{-} \mathbf{M}\right)$ the set of vertices in $\mathbf{V}^{+}$ (resp. in $\mathbf{V}^{-}$) covered by the edges of $M$ and we note $\mathbf{S}_{\mathbf{0}}^{+}=\mathbf{V}^{+} \backslash \partial^{+} \mathbf{M}$. Then, we use the following algorithm:

$\leftrightarrow$ Find a maximal matching $M$ in $B\left(\Sigma_{\Lambda}\right)$,

$\leftrightarrow \overline{\mathbf{V}}_{\mathbf{0}}^{+}=\mathbf{S}_{\mathbf{0}}^{+} \cup\left\{\mathbf{v} \in \mathbf{V}^{+}, \exists\right.$ a path in $B_{M}\left(\Sigma_{\Lambda}\right)$ from $\mathbf{S}_{\mathbf{0}}^{+}$to $\left.\mathbf{v}\right\}$ $\leftrightarrow \mathbf{V}_{\mathbf{0}}^{+}=\overline{\mathbf{V}}_{\mathbf{0}}^{+} \backslash\left\{\mathbf{v} \in \overline{\mathbf{V}}_{\mathbf{0}}^{+} \cap \overline{\mathbf{U}}^{+}, \nexists\right.$ a path in $B_{M}\left(\Sigma_{\Lambda}\right)$ from $\mathbf{S}_{\mathbf{0}}^{+} \cap$ $\mathbf{X}_{\mathbf{i}}^{+}$to $\mathbf{v}$ or from $\mathbf{v}$ to $\left.\mathbf{S}_{\mathbf{0}}^{+} \cap \mathbf{X}_{\mathbf{i}}^{+}\right\}$

$\leftrightarrow \mathbf{V}_{\mathbf{0}}^{-}=\left\{\mathbf{v} \in \mathbf{V}^{-}, \exists\right.$ a path in $B_{M}\left(\Sigma_{\Lambda}\right)$ from $\mathbf{V}_{\mathbf{0}}^{+}$to $\left.\mathbf{v}\right\}$ It is important to note that the obtained subsets $\mathbf{V}_{\mathbf{0}}^{+}$and $\mathbf{V}_{\mathbf{0}}^{-}$ are the same whatever the choice of the maximal matching $M$ (Dulmage and Mendelsohn [1958], Murota [1987]). Using the previous algorithm, we have:

Proposition 4. Consider structured system $\Sigma_{\Lambda}$ represented by digraph $\mathcal{G}\left(\Sigma_{\Lambda}\right)$ and constituted by subsystems $\Sigma_{i}^{R}, i=$ $1, \ldots, N$. To recover the maximal matching condition, for subsystem $\Sigma_{i}^{R}$ the minimal number of additional measures transmitted by the network, noted $\tilde{\mathbf{Y}}_{\mathbf{i}}$, is equal to $\gamma=\operatorname{card}\left(\mathbf{V}_{\mathbf{0}}^{+}\right)-$ card $\left(\mathbf{V}_{\mathbf{0}}^{-}\right)$. These additional measurements $\gamma$ concern unknown inputs in $\mathbf{V}_{\mathbf{0}}^{+} \cap \tilde{\mathbf{U}}_{\mathbf{i}}^{+}$such that we obtain a maximal matching of size card $\left(\mathbf{V}_{\mathbf{0}}^{+}\right)$between $\mathbf{V}_{\mathbf{0}}^{+}$and $\mathbf{V}_{\mathbf{0}}^{-} \cup \tilde{\mathbf{Y}}_{\mathbf{i}}$.
Proof: the proof is similar to the one given in Commault et al. [2008], Boukhobza and Hamelin [2009].

Let us now illustrate the previous settings by considering Subsystem 1 of Example 3 . The bipartite graph associated to such system is presented in Figure 2.

$\uparrow$ Select a maximal matching $M$ in this bipartite graph, which

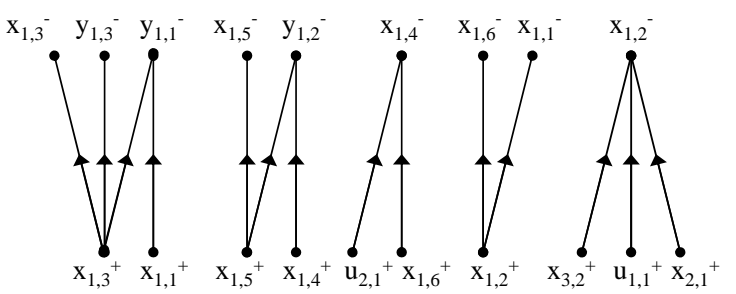

Figure 2. Bipartite graph associated to Subsystem 1 of Example 3

can be: $M=\left\{\left(\mathbf{x}_{1,3}^{+}, \mathbf{x}_{1,3}^{-}\right),\left(\mathbf{x}_{1, \mathbf{1}}^{+}, \mathbf{y}_{1, \mathbf{1}}^{-}\right),\left(\mathbf{x}_{1, \mathbf{5}}^{+}, \mathbf{x}_{1,5}^{-}\right),\left(\mathbf{x}_{1,4}^{+}, \mathbf{y}_{\mathbf{1 , 2}}^{-}\right)\right.$,

$\left.\left(\mathbf{u}_{2,1}^{+}, \mathbf{x}_{1,4}^{-}\right),\left(\mathbf{x}_{1,2}^{+}, \mathbf{x}_{1,6}^{-}\right),\left(\mathbf{x}_{3,2}^{+}, \mathbf{x}_{1,2}^{-}\right)\right\}$. In this case,

$\mathbf{S}_{\mathbf{0}}^{+}=\left\{\mathbf{x}_{1,6}^{+}, \mathbf{u}_{1,1}^{+}, \mathbf{x}_{2,1}^{+}\right\}$.

$\leftrightarrow \overline{\mathbf{V}}_{\mathbf{0}}^{+}=\left\{\mathbf{u}_{\mathbf{2}, \mathbf{1}}^{+}, \mathbf{x}_{\mathbf{1}, \mathbf{6}}^{+}, \mathbf{x}_{\mathbf{3}, \mathbf{2}}^{+}, \mathbf{u}_{\mathbf{1}, \mathbf{1}}^{+}, \mathbf{x}_{\mathbf{2}, \mathbf{1}}^{+}\right\}$

$\leftrightarrow \mathbf{V}_{\mathbf{0}}^{+}=\left\{\mathbf{u}_{\mathbf{2}, \mathbf{1}}^{+}, \mathbf{x}_{\mathbf{1}, \mathbf{6}}^{+}\right\}$.

$\rightarrow \mathbf{V}_{\mathbf{0}}^{-}=\left\{\mathbf{x}_{\mathbf{1}, \mathbf{4}^{-}}\right\}$.

Since card $\left(\mathbf{V}_{\mathbf{0}}^{+}\right)-\operatorname{card}\left(\mathbf{V}_{\mathbf{0}}^{-}\right)=1$, only one transmitted measurement is sufficient to guarantee the maximal matching condition. Moreover, the only possibility is to transmit $\mathbf{u}_{\mathbf{2}, \mathbf{1}}$ as it is the only external variable to Subsystem 1 in $\mathbf{V}_{\mathbf{0}}^{+}$. Note that in this case, the length condition has been also recovered by the transmission of $\mathbf{u}_{2,1}$ even if this subsystem is submitted to other external inputs.

It can be useful to specify more precisely the additional measurements transmitted to the considered subsystem to recover the I.C. maximal matching condition. At this aim, let us define by $\bar{B}\left(\Sigma_{\Lambda}\right)$ the non-directed graph corresponding to $B\left(\Sigma_{\Lambda}\right)$ and $\mathbf{V}_{\mathbf{0}} \stackrel{\text { def }}{=} \mathbf{V}_{\mathbf{0}}^{+} \cup \mathbf{V}_{\mathbf{0}}^{-}$. We call $\mathbf{V}_{\mathbf{0}}$-path every simple path of $\bar{B}\left(\Sigma_{\Lambda}\right)$ which covers only vertices of $\mathbf{V}_{\mathbf{0}}$. For each $\mathbf{V}_{\mathbf{0}}$-path $P$, we define a vertex subset $\vartheta(P)$ such that $\vartheta(P)=\{\mathbf{v} \in$ $V_{0}^{+}$, such that $P$ covers $\left.\mathbf{v}\right\}$. Finally, we say that a $\mathbf{V}_{\mathbf{0}}$-path $P$ is maximal, if there does not exist a $\mathbf{V}_{\mathbf{0}}$-path $P^{\prime}$ such that $\vartheta(P) \subset \vartheta\left(P^{\prime}\right)$. To recover the maximal matching condition, it is necessary to have:

$$
\text { for each maximal } \mathbf{V}_{\mathbf{0}} \text {-path } P, \theta\left(\vartheta(P), \tilde{\mathbf{Y}}_{\mathbf{i}}^{-}\right) \neq 0
$$

Moreover to recover the maximal matching condition, it is necessary to have, for each maximal $\mathbf{V}_{\mathbf{0}}$-path $P$ :

$$
\theta\left(\mathbf{V}_{\mathbf{0}}^{+}, \tilde{\mathbf{Y}}_{\mathbf{i}}^{-} \cup \mathbf{V}_{\mathbf{0}}^{-}\right)-\theta\left(\mathbf{V}_{\mathbf{0}}^{+} \backslash \vartheta(P), \tilde{\mathbf{Y}}_{\mathbf{i}}^{-} \cup \mathbf{V}_{\mathbf{0}}^{-}\right)>0
$$

Conditions (5) and (6) ensure that there is at least one measurement in each subset $\vartheta(P) \cap \tilde{\mathbf{U}}_{\mathbf{i}}$. Indeed, $\vartheta(P)$ satisfy, by construction $\theta\left(\vartheta(P), \mathbf{V}_{\mathbf{0}}^{-}\right)=\operatorname{card}(\vartheta(P))-1$ and so necessitates the transmission of one measurement to recover the maximal matching condition.

\subsection{Transmitted external measurements for the I.C. length condition}

Assume that condition Cond1. of Proposition 3 is now verified. Hence, we may have transmit some measurements to subsystem $\Sigma_{i}^{R}$ to the system in order to satisfy I.C. maximal matching condition. In this case, we can consider in the sequel that all the vertices associated to the measured external inputs of $\Sigma_{i}^{R}$ are removed from $\overline{\mathbf{U}}_{\mathbf{i}}$ and from the digraph as well as all the 
edges beginning by these vertices.

Let us denote by $\boldsymbol{\Delta}_{\mathbf{i}} \stackrel{\text { def }}{=}\left\{\mathbf{x}_{\ell} \in \mathbf{X}_{\mathbf{i}} \mid \rho\left(\overline{\mathbf{U}}_{\mathbf{i}} \cup\left\{\mathbf{x}_{\ell}\right\}, \mathbf{Y}_{\mathbf{i}}\right)=\right.$ $\left.\rho\left(\overline{\mathbf{U}}_{\mathbf{i}}, \mathbf{Y}_{\mathbf{i}}\right)\right\}$. If Condition Cond1. of Proposition 3 is satisfied then it is easy to see that $\rho\left(\overline{\mathbf{U}}_{\mathbf{i}}, \mathbf{Y}_{\mathbf{i}}\right)=\theta\left(\overline{\mathbf{U}}_{\mathbf{i}}, \mathbf{Y}_{\mathbf{i}}\right)$. We can deduce that, whatever the transmitted measurements represented by vertex subset $\tilde{\mathbf{Y}}_{\mathbf{i}}$, we have $V_{\text {ess }}\left(\overline{\mathbf{U}}_{\mathbf{i}}, \mathbf{Y}_{\mathbf{i}} \cup \tilde{\mathbf{Y}}_{\mathbf{i}}\right) \cap$ $\mathbf{X}_{\mathbf{i}} \subseteq V_{\text {ess }}\left(\overline{\mathbf{U}}_{\mathbf{i}}, \mathbf{Y}_{\mathbf{i}}\right) \cap \mathbf{X}_{\mathbf{i}}$. Consequently, if an element $\mathbf{x}_{\ell}$ is in $\boldsymbol{\Delta}_{\mathbf{i}}$ but not in $V_{\text {ess }}\left(\overline{\mathbf{U}}_{\mathbf{i}}, \mathbf{Y}_{\mathbf{i}}\right)$, then adding measures $\tilde{\mathbf{Y}}_{\mathbf{i}}$ anywhere on $\tilde{\mathbf{U}}_{\mathbf{i}}$, we cannot obtain that $\mathbf{x}_{\mathbf{i}} \in V_{\text {ess }}\left(\overline{\mathbf{U}}_{\mathbf{i}}, \mathbf{Y}_{\mathbf{i}} \cup \tilde{\mathbf{Y}}_{\mathbf{i}}\right)$. Hence, the only way to ensure condition Cond3. of Proposition 3 is to remove from $\boldsymbol{\Delta}_{\mathbf{i}}$ all the elements which do not belong to $V_{e s s}\left(\overline{\mathbf{U}}_{\mathbf{i}}, \mathbf{Y}_{\mathbf{i}}\right)$.

According to this fact, to recover condition Cond3. of Proposition 3 , it is necessary and sufficient to transmit some measurements $\tilde{\mathbf{Y}}_{\mathbf{i}}$, such that, for each $\mathbf{x}_{\ell} \in \boldsymbol{\Delta}_{\mathbf{i}} \backslash V_{\text {ess }}\left(\overline{\mathbf{U}}_{\mathbf{i}}, \mathbf{Y}_{\mathbf{i}}\right)$, we have $\rho\left(\overline{\mathbf{U}}_{\mathbf{i}} \cup\left\{\mathbf{x}_{\ell}\right\}, \mathbf{Y}_{\mathbf{i}} \cup \tilde{\mathbf{Y}}_{\mathbf{i}}\right)>\rho\left(\overline{\mathbf{U}}_{\mathbf{i}}, \mathbf{Y}_{\mathbf{i}} \cup \tilde{\mathbf{Y}}_{\mathbf{i}}\right)$. Considering virtually $\mathbf{x}_{\ell}$ as an input vertex, a necessary and sufficient condition to guarantee inequality $\rho\left(\overline{\mathbf{U}}_{\mathbf{i}} \cup\left\{\mathbf{x}_{\ell}\right\}, \mathbf{Y}_{\mathbf{i}} \cup \tilde{\mathbf{Y}}_{\mathbf{i}}\right)>\rho\left(\overline{\mathbf{U}}_{\mathbf{i}}, \mathbf{Y}_{\mathbf{i}} \cup\right.$ $\left.\tilde{\mathbf{Y}}_{\mathbf{i}}\right)$ is that the added measurements $\tilde{\mathbf{Y}}_{\mathbf{i}}$ concern only input vertices covered by any direct $\overline{\mathbf{U}}_{\mathbf{i}} \cup\left\{\mathbf{x}_{\ell}\right\}-\mathbf{S}^{\mathbf{i}}\left(\overline{\mathbf{U}}_{\mathbf{i}} \cup\left\{\mathbf{x}_{\mathbf{i}}\right\}, \mathbf{Y}_{\mathbf{i}}\right)$ path which has a nonzero length (Commault and Dion [2007]). But there can exist some edges arriving to $\mathbf{x}_{\ell}$ since the latter is not really an input vertex. Thus, the only other measurements which can guarantee inequality $\rho\left(\overline{\mathbf{U}}_{\mathbf{i}} \cup\left\{\mathbf{x}_{\ell}\right\}, \mathbf{Y}_{\mathbf{i}} \cup \tilde{\mathbf{Y}}_{\mathbf{i}}\right)>$ $\rho\left(\overline{\mathbf{U}}_{\mathbf{i}}, \mathbf{Y}_{\mathbf{i}} \cup \tilde{\mathbf{Y}}_{\mathbf{i}}\right)$ are also the ones done on any direct $\overline{\mathbf{U}}_{\mathbf{i}} \cup\left\{\mathbf{x}_{\mathbf{k}}\right\}$ $\mathbf{S}^{\mathbf{i}}\left(\overline{\mathbf{U}}_{\mathbf{i}} \cup\left\{\mathbf{x}_{\mathbf{i}}\right\}, \mathbf{Y}_{\mathbf{i}}\right)$ where $\mathbf{x}_{\mathbf{k}}$ is such that $\rho\left(\left\{\mathbf{x}_{\mathbf{k}}\right\},\left\{\mathbf{x}_{\ell}\right\}\right)=$ $\rho\left(\left\{\mathbf{x}_{\ell}\right\},\left\{\mathbf{x}_{\mathbf{k}}\right\}\right)=1$. Finally, since $\tilde{\mathbf{Y}}_{\mathbf{i}}$ represent measurements transmitted via the network concerning other subsystems, they concern only the unknown input vertices $\tilde{\mathbf{U}}_{\mathbf{i}}$. Thus, considering $\mathbf{x}_{\ell}$ or all the $\mathbf{x}_{\mathbf{k}}$ such that $\rho\left(\left\{\mathbf{x}_{\mathbf{k}}\right\},\left\{\mathbf{x}_{\ell}\right\}\right)=\rho\left(\left\{\mathbf{x}_{\ell}\right\},\left\{\mathbf{x}_{\mathbf{k}}\right\}\right)=1$, the elements of $\tilde{\mathbf{U}}_{\mathbf{i}}$ which covered by any direct $\overline{\mathbf{U}}_{\mathbf{i}} \cup\left\{\mathbf{x}_{\ell}\right\}$ $\mathbf{S}^{\mathbf{i}}\left(\overline{\mathbf{U}}_{\mathbf{i}} \cup\left\{\mathbf{x}_{\mathbf{i}}\right\}, \mathbf{Y}_{\mathbf{i}}\right)$ path are the same than the ones covered by any direct $\overline{\mathbf{U}}_{\mathbf{i}} \cup\left\{\mathbf{x}_{\mathbf{k}}\right\}-\mathbf{S}^{\mathbf{i}}\left(\overline{\mathbf{U}}_{\mathbf{i}} \cup\left\{\mathbf{x}_{\mathbf{i}}\right\}, \mathbf{Y}_{\mathbf{i}}\right)$ path. Thus, it is necessary and sufficient to consider virtually all the vertices belonging to $\boldsymbol{\Delta}_{\mathbf{i}} \backslash V_{\text {ess }}\left(\overline{\mathbf{U}}_{\mathbf{i}}, \mathbf{Y}_{\mathbf{i}}\right)$ as input vertices.

Let us define, for each $\mathbf{x}_{\ell} \in \boldsymbol{\Delta}_{\mathbf{i}} \backslash V_{\text {ess }}\left(\overline{\mathbf{U}}_{\mathbf{i}}, \mathbf{Y}_{\mathbf{i}}\right)$, vertex subset $\delta_{\ell} \stackrel{\text { def }}{=}\left\{\mathbf{u}_{\mathbf{j}} \in \tilde{\mathbf{U}}_{\mathbf{i}}, \mathbf{u}_{\mathbf{j}}\right.$ is the begin vertex of some direct $\overline{\mathbf{U}}_{\mathbf{i}} \cup$ $\left\{\mathbf{x}_{\ell}\right\}-\mathbf{S}^{\mathbf{i}}\left(\overline{\mathbf{U}}_{\mathbf{i}} \cup\left\{\mathbf{x}_{\mathbf{i}}\right\}, \mathbf{Y}_{\mathbf{i}}\right)$ path, such that $\rho\left(\overline{\mathbf{U}}_{\mathbf{i}} \cup\left\{\mathbf{x}_{\ell}\right\}, \mathbf{Y}_{\mathbf{i}} \cup\right.$ $\left.\left.\tilde{\mathbf{Y}}_{\mathbf{i}}\right)>\rho\left(\overline{\mathbf{U}}_{\mathbf{i}} \backslash\left\{\mathbf{u}_{\mathbf{j}}\right\}, \mathbf{Y}_{\mathbf{i}}\right)\right\}$. We can state:

Proposition 5. Consider structured system $\Sigma_{\Lambda}$ represented by digraph $\mathcal{G}\left(\Sigma_{\Lambda}\right)$ and constituted by subsystems $\Sigma_{i}^{R}, i=$ $1, \ldots, N$. Assume that each subsystem is strongly locally observable. To recover the I.C. length condition of Proposition 3 , for subsystem $\Sigma_{i}^{R}$, the measurements transmitted by the network, noted $\tilde{\mathbf{Y}}_{\mathbf{i}}$ must concern all the external components in $\mathbf{V}_{\mathbf{U}} \stackrel{\text { def }}{=} \bigcup_{\mathbf{x}_{\ell} \in \boldsymbol{\Delta}_{\mathbf{i}} \backslash V_{e s s}\left(\overline{\mathbf{U}}_{\mathbf{i}}, \mathbf{Y}_{\mathbf{i}}\right)} \delta_{\ell}$ such that $\mathbf{V}_{\mathbf{U}} \subseteq \mathbf{S}^{\mathbf{o}}\left(\overline{\mathbf{U}}_{\mathbf{i}}, \mathbf{Y}_{\mathbf{i}} \cup \tilde{\mathbf{Y}}_{\mathbf{i}}\right)$.

Proof: The proof is immediate from the previous settings. $\triangle$

\section{CONCLUSION}

An important problem that must be considered when dealing with control over network, is the validity of some properties as the observability. For network distributed systems, an alternative to the centralized observation scheme, which can be quite complicated to realize when we deal with a large scale system, is to consider a decentralized distributed observation scheme or a completely autonomous observation scheme. The first scheme corresponds to the case when the subsystem is connected to the network and receive some informations from the other subsystems. The second scheme is related to the case when the subsystem have only its own measurements to reconstruct a part of the state and input components as in a network cut for example. In this paper, we propose first an analysis tool to study the generic strong observability of network distributed structured linear systems in both distributed interconnected and distributed autonomous schemes. In a second part of the paper, we give a procedure of measurement distribution through the network. More precisely, for any given subsystem $\Sigma_{i}^{R}$, we indicate quite precisely, which external measurement must be transmitted through the network from the other subsystem in order to ensure the strong observability of $\Sigma_{i}^{R}$ in a decentralized interconnected observation scheme.

Our approach is based on a graph-theoretic approach,and needs few information about the system. The provide results are very easy to check by means of well-known combinatorial techniques and simply by hand for small systems. Indeed, from a computational point of view, our proposed approach needs few information about the system and is quite easy to check by means of well-known combinatorial techniques or simply by hand for small systems. Indeed, it uses classical programming techniques like Ford-Fulkerson algorithm to compute the input separators in a digraph and Dulmage-Mendelsohn decomposition of a bipartite graph. These algorithms are free from numerical difficulties and lead polynomial complexity order implementations.

\section{REFERENCES}

T. Boukhobza and F. Hamelin. State and input observability recovering by additional sensor implementation: a graph-theoretic approach. Automatica, 45(7):1737-1742, 2009.

T. Boukhobza, F. Hamelin, and S. Martinez-Martinez. State and input observability for structured linear Systems: a graph-theoretic approach. Automatica, 43(7):1204-1210, 2007.

C. Commault and J. M. Dion. Sensor location for diagnosis in linear Systems: a structural analysis. IEEE Transactions on Automatic Control, 52(2):155$169,2007$.

C. Commault, J-M. Dion, and D. H. Trinh. Observability preservation under sensor failure. IEEE Transactions on Automatic Control, 53(6):1554-1559, 2008.

J. M. Dion, C. Commault, and J. W. van der Woude. Generic properties and control of linear structured systems: a survey. Automatica, 39(7):11251144, 2003.

A. L. Dulmage and N. S. Mendelsohn. Coverings of bipartite graphs. Canadian Journal of Mathematics, 10:517-534, 1958.

M. Hou and P. C. Müller. Fault detection and isolation observers. International Journal of Control, 60(5):827-846, 1994.

K. Murota. System Analysis by Graphs and Matroids. Springer-Verlag, New York, U.S.A., 1987.

M. Saif and Y. Guan. Decentralized state estimation in large-scale interconnected dynamical systems. Automatica, 28(1):215-219, 1992.

C. W. Sanders, E. C. Tacker, and T. D. Lindon. A new class of decentralized filters for interconnected systems. IEEE Transactions on Automatic Control, AC-23(2):259-262, 1974.

H. L. Trentelman, A. A. Stoorvogel, and M. Hautus. Control Theory for Linear Systems. Springer, London, U.K., 2001. 